\title{
Further Results on Uniqueness of Meromorphic Functions concerning Fixed Points
}

\author{
Xiao-Bin Zhang \\ College of Science, Civil Aviation University of China, Tianjin 300300, China \\ Correspondence should be addressed to Xiao-Bin Zhang; tclzxb@163.com \\ Received 30 April 2014; Accepted 29 July 2014; Published 16 October 2014 \\ Academic Editor: Adrian Petrusel
}

Copyright (C) 2014 Xiao-Bin Zhang. This is an open access article distributed under the Creative Commons Attribution License, which permits unrestricted use, distribution, and reproduction in any medium, provided the original work is properly cited.

We will study the uniqueness problems of meromorphic functions of differential polynomials sharing fixed points. Our results improve or generalize some previous results on meromorphic functions sharing fixed points.

\section{Introduction and Main Results}

Let $\mathbb{C}$ denote the complex plane and let $f(z)$ be a nonconstant meromorphic function on $\mathbb{C}$. We assume the reader is familiar with the standard notion used in the Nevanlinna value distribution theory such as $T(r, f), m(r, f)$, and $N(r, f)$ (see, e.g., [1-3]), and $S(r, f)$ denotes any quantity that satisfies the condition $S(r, f)=o(T(r, f))$ as $r \rightarrow \infty$ outside of a possible exceptional set of finite linear measure.

Let $f(z)$ and $g(z)$ be two nonconstant meromorphic functions. Let $a \in \mathbb{C} \bigcup\{\infty\}$; we say that $f(z), g(z)$ share $a \mathrm{CM}$ (counting multiplicities) if $f(z)-a, g(z)-a$ have the same zeros with the same multiplicities and we say that $f(z), g(z)$ share $a$ IM (ignoring multiplicities) if we do not consider the multiplicities. We denote by $\bar{N}_{L}(r, 1 /(f-a))\left(\bar{N}_{L}(r, 1 /(g-\right.$ $a))$ ) the counting function of those $a$-points of $f$ whose multiplicities are greater (less) than the multiplicities of the corresponding $a$-points of $g$, where each $a$-point is counted only once. $N_{k}(r, 1 /(f-a))$ denotes the truncated counting function bounded by $k$.

We say that a finite value $z_{0}$ is called a fixed point of $f$ if $f\left(z_{0}\right)=z_{0}$ or $z_{0}$ is a zero of $f(z)-z$.

The following theorem in the value distribution theory is well-known $[4,5]$.

Theorem A. Let $f(z)$ be a transcendental meromorphic function and $n \geq 1$ a positive integer. Then $f^{n} f^{\prime}=1$ has infinitely many solutions.
Related to Theorem A, Fang [6] proved that a meromorphic function $f^{n} f^{\prime}$ has infinitely many fixed points when $f$ is transcendental and $n$ is a positive integer. Then Fang and Qiu [7] obtained the following uniqueness theorem.

Theorem B. Let $f$ and $g$ be two nonconstant meromorphic (entire) functions and $n \geq 11(\geq 6)$ a positive integer. If $f^{n}(z) f^{\prime}(z)$ and $g^{n}(z) g^{\prime}(z)$ share $z C M$, then either $f(z)=$ $c_{1} e^{c z^{2}}, g(z)=c_{2} e^{-c z^{2}}$, where $c_{1}, c_{2}$, and $c$ are three constants satisfying $4\left(c_{1} c_{2}\right)^{n+1} c^{2}=-1$, or $f(z) \equiv \operatorname{tg}(z)$ for a constant $t$ such that $t^{n+1}=1$.

For more related results, see [8,9]. Recently, Cao and Zhang [10] replaced $f^{\prime}$ with $f^{(k)}$ and obtained the following.

Theorem C. Let $f(z)$ and $g(z)$ be two transcendental meromorphic functions, whose zeros are of multiplicities at least $k$, where $k$ is a positive integer; let $n>\max \{2 k-1, k+4 / k+4\}$ be a positive integer. If $f^{n} f^{(k)}$ and $g^{n} g^{(k)}$ share $z C M$, and $f$ and $g$ share $\infty I M$, then one of the following two conclusions holds:

(1) $f^{n} f^{(k)}=g^{n} g^{(k)}$;

(2) $f=c_{1} e^{c z^{2}}, g=c_{2} e^{-c z^{2}}$, where $c_{1}, c_{2}$, and c are constants such that $4\left(c_{1} c_{2}\right)^{n+1} c^{2}=-1$.

Regarding Theorem C, it is natural to ask the following. 
Problem 1. Does Theorem C still hold without the "transcendental" condition?

Problem 2. Does Theorem C still hold without the "multiplicity of zeros of $f$ and $g$ " condition?

Problem 3. Can the lower bound of $n$ be reduced in Theorem C?

We consider Problems 1-3 and give affirmative answers to them, and we get the following.

Theorem 1. Let $f(z)$ and $g(z)$ be two nonconstant meromorphic functions and $n, k$ two positive integers with $n>k+8$. If $f^{n} f^{(k)}$ and $g^{n} g^{(k)}$ share $z C M$, and $f$ and $g$ share $\infty I M$, then one of the following two conclusions holds:

(1) $f^{n} f^{(k)}=g^{n} g^{(k)}$;

(2) $f=c_{1} e^{c z^{2}}, g=c_{2} e^{-c z^{2}}$, where $c_{1}, c_{2}$, and $c$ are constants such that $4\left(c_{1} c_{2}\right)^{n+1} c^{2}=-1$.

One may ask whether the condition " $n>k+8$ " can be further reduced. We have proved the following.

Theorem 2. Let $f(z)$ and $g(z)$ be two nonconstant meromorphic functions and $n, k$ two positive integers with $n>k+7$. If $f^{n} f^{(k)}$ and $g^{n} g^{(k)}$ share $z C M$, and $f$ and $g$ share $\infty I M$, then one of the following two conclusions holds:

(1) $f^{n} f^{(k)}=g^{n} g^{(k)}$, possibly except for at most one exceptional case, namely,

$$
\begin{gathered}
k=1, \quad n=9, \\
f=a \frac{\left(z-a_{1}\right)\left(z-a_{2}\right)\left(z-a_{3}\right)}{\left(z-d_{1}\right)^{2}\left(z-d_{2}\right)}, \\
g=b \frac{\left(z-b_{1}\right)\left(z-b_{2}\right)\left(z-b_{3}\right)}{\left(z-d_{1}\right)\left(z-d_{2}\right)^{2}}
\end{gathered}
$$

where $a_{j}, b_{j}(j=1,2,3), d_{1}, d_{2}$ are 8 distinct constants and $a, b$ are two nonzero constants;

(2) $f=c_{1} e^{c z^{2}}, g=c_{2} e^{-c z^{2}}$, where $c_{1}, c_{2}$, and $c$ are constants such that $4\left(c_{1} c_{2}\right)^{n+1} c^{2}=-1$.

If $f$ and $g$ are two transcendental meromorphic functions, the lower bound of $n$ in Theorem 2 can be further reduced. We have the following.

Theorem 3. Let $f(z)$ and $g(z)$ be two transcendental meromorphic functions and $n, k$ two positive integers with $n>k+6$. If $f^{n} f^{(k)}$ and $g^{n} g^{(k)}$ share $z C M$, and $f$ and $g$ share $\infty I M$, then one of the following two conclusions holds:

(1) $f^{n} f^{(k)}=g^{n} g^{(k)}$;

(2) $f=c_{1} e^{c z^{2}}, g=c_{2} e^{-c z^{2}}$, where $c_{1}, c_{2}$, and c are constants such that $4\left(c_{1} c_{2}\right)^{n+1} c^{2}=-1$.

\section{Preliminary Lemmas}

Let

$$
\begin{aligned}
& H=\left(\frac{F^{\prime \prime}}{F^{\prime}}-\frac{2 F^{\prime}}{F-1}\right)-\left(\frac{G^{\prime \prime}}{G^{\prime}}-\frac{2 G^{\prime}}{G-1}\right), \\
& V=\left(\frac{F^{\prime}}{F-1}-\frac{F^{\prime}}{F}\right)-\left(\frac{G^{\prime}}{G-1}-\frac{G^{\prime}}{G}\right),
\end{aligned}
$$

where $F$ and $G$ are meromorphic functions.

Lemma 4 (see [11]). Let $f(z)$ be a nonconstant meromorphic function and let $a_{0}(z), a_{1}(z), \ldots, a_{n}(z)(\not \equiv)$ be small functions with respect to $f$. Then

$$
T\left(r, a_{n} f^{n}+a_{n-1} f^{n-1}+\cdots+a_{0}\right)=n T(r, f)+S(r, f) .
$$

Lemma 5 (see [2]). Let $f(z)$ be a nonconstant meromorphic function, and let $k$ be a positive integer. Suppose that $f^{(k)} \neq 0$; then

$$
N\left(r, \frac{1}{f^{(k)}}\right) \leq N\left(r, \frac{1}{f}\right)+k \bar{N}(r, f)+S(r, f) .
$$

By using the similar method to Yang and Hua [12, Lemma 3], we can prove the following lemma.

Lemma 6. Let $F, G$, and $H$ be defined as in (2). If $F$ and $G$ share $1 C M$ and $\infty I M$, and $H \neq \equiv 0$, then $F \neq \equiv$, and

$$
\begin{aligned}
T(r, F) \leq & N_{2}\left(r, \frac{1}{F}\right)+N_{2}\left(r, \frac{1}{G}\right)+2 \bar{N}(r, F) \\
& +\bar{N}_{L}(r, F)+\bar{N}_{L}(r, G)+S(r, F)+S(r, G),
\end{aligned}
$$

the same inequality holding for $T(r, G)$.

Lemma 7 (see [13]). Let $F, G$, and $V$ be defined as in (3). If $F$ and $G$ share $\infty I M$, and $V \equiv 0$, then $F \equiv G$.

Lemma 8. Let $f, g$ be two nonconstant meromorphic functions, $V$ defined as in (3), where $F=f^{n} f^{(k)} / z, G=$ $g^{n} g^{(k)} / z$, and $n>0, k>0$, and $m \geq 0$ three integers. If $V \neq \equiv$ $0, F$ and $G$ share $1 C M$, and $f$ and $g$ share $\infty I M$, then

$$
\begin{aligned}
(n-k) \bar{N}(r, f)= & (n-k) \bar{N}(r, g) \\
\leq & 2\left(N\left(r, \frac{1}{f}\right)+N\left(r, \frac{1}{g}\right)\right) \\
& +S(r, f)+S(r, g) .
\end{aligned}
$$

Proof. Note that $V \not \equiv 0, f$ and $g$ share $\infty$ IM, suppose that $z_{0} \neq 0$ is a pole of $f(g)$ with multiplicity $p(q)$, then $z_{0}$ is a pole of $F(G)$ with multiplicity $n p+p+k(n q+q+k)$. Thus $z_{0}$ is a zero of $F^{\prime} /(F-1)-F^{\prime} / F$ with multiplicity $n p+p+k-1(\geq n+k)$, and a zero of $G^{\prime} /(G-1)-G^{\prime} / G$ with multiplicity $n p+p+k-1(\geq$ $n+k)$. Hence $z_{0}$ is a zero of $V$ with multiplicity at least $n+k$. Suppose that $z_{1}=0$ is a pole of $f(g)$ with multiplicity $r(s)$, by 
the similar discussion as above, we get that $z_{1}=0$ is a zero of $V$ with multiplicity at least $n+k+1$. So we have

$$
(n+k) \bar{N}(r, f)=(n+k) \bar{N}(r, g) \leq N\left(r, \frac{1}{V}\right) .
$$

By the logarithmic derivative lemma, we have $m(r, V)=$ $S(r, f)+S(r, g)$. Note that $F$ and $G$ share $1 \mathrm{CM}$, so we have

$$
\begin{aligned}
N\left(r, \frac{1}{V}\right) & \leq T(r, V)=m(r, V)+N(r, V) \\
& \leq \bar{N}\left(r, \frac{1}{F}\right)+\bar{N}\left(r, \frac{1}{G}\right)+S(r, f)+S(r, g) .
\end{aligned}
$$

Obviously,

$$
\begin{aligned}
& \bar{N}\left(r, \frac{1}{F}\right) \leq 2 N\left(r, \frac{1}{f}\right)+k \bar{N}(r, f)+S(r, f), \\
& \bar{N}\left(r, \frac{1}{G}\right) \leq 2 N\left(r, \frac{1}{g}\right)+k \bar{N}(r, g)+S(r, g) .
\end{aligned}
$$

From (8)-(10) we get (7). This proves Lemma 8.

Lemma 9 (see [1, Theorem 3.10]). Suppose that $f$ is a nonconstant meromorphic function; $k \geq 2$ is an integer. If

$$
N(r, f)+N\left(r, \frac{1}{f}\right)+N\left(r, \frac{1}{f^{(k)}}\right)=S\left(r, \frac{f^{\prime}}{f}\right)
$$

then $f=e^{a z+b}$, where $a \neq 0, b$ are constants.

Lemma 10. Let $f, g$ be two nonconstant meromorphic functions and $n(\geq 2), k$ two positive integers. If $f^{n} f^{(k)} g^{n} g^{(k)}=z^{2}$, and $f$ and $g$ share $\infty$ IM, then $f=c_{1} e^{c z^{2}}, g=c_{2} e^{-c z^{2}}$, where $c_{1}$, $c_{2}$, and $c$ are constants such that $4\left(c_{1} c_{2}\right)^{n+1} c^{2}=-1$.

Proof. Since $f$ and $g$ share $\infty$ IM, from

$$
f^{n} f^{(k)} g^{n} g^{(k)}=z^{2}
$$

we get that both $f$ and $g$ are entire functions.

The case $k=1$ has been proved by Fang and Qiu [7, Propostion 2]; here we only need to consider the case $k \geq 2$.

Let $F_{1}=f^{n} f^{(k)}, G_{1}=g^{n} g^{(k)}$. Then we have

$$
\begin{aligned}
n T(r, f) & =T\left(r, f^{n}\right) \\
& =T\left(r, \frac{F_{1}}{f^{(k)}}\right) \\
& \leq T\left(r, F_{1}\right)+T\left(r, f^{(k)}\right)+S(r, f) \\
& \leq T\left(r, F_{1}\right)+T(r, f)+S(r, f) .
\end{aligned}
$$

We obtain from (13) that

$$
T(r, f)=O\left(T\left(r, F_{1}\right)\right)
$$

Note that

$$
\begin{aligned}
T\left(r, F_{1}\right) & =T\left(r, f^{n} f^{(k)}\right) \\
& \leq n T(r, f)+T\left(r, f^{(k)}\right)+S(r, f) \\
& \leq(n+1) T(r, f)+S(r, f) .
\end{aligned}
$$

We obtain from (15) that

$$
T(r, F)=O(T(r, f)) .
$$

Thus from (14) and (16) we have $\sigma(f)=\sigma(F)$. Similarly we have $\sigma(g)=\sigma(G)$. It follows from (12) that $\sigma(F)=\sigma(G)$; we get $\sigma(f)=\sigma(g)$.

Suppose that $f$ has a zero $z_{0}$, say multiplicity $p$; then $z_{0}$ is a zero of $f^{n}$ with multiplicity $n p \geq 2$. In view of (12), we get $n=2$ and $z_{0}=0$. Moreover, $g$ has no zero. Therefore,

$$
f(z)=z e^{\alpha_{1}(z)}, \quad g(z)=e^{\beta_{1}(z)},
$$

where $\alpha_{1}(z), \beta_{1}(z)$ are nonconstant entire functions. We deduce that either both $\alpha$ and $\beta$ are transcendental functions or both $\alpha_{1}$ and $\beta_{1}$ are polynomials. From (17) we have

$$
T\left(r, \frac{f^{\prime}}{f}\right)=T\left(r, \alpha_{1}^{\prime}+\frac{1}{z}\right) .
$$

Moreover, we have

$$
N\left(r, \frac{1}{f^{(k)}}\right)=0 \text {. }
$$

Thus we get

$$
N(r, f)+N\left(r, \frac{1}{f}\right)+N\left(r, \frac{1}{f^{(k)}}\right)=O(\log r) .
$$

If $k \geq 2$, suppose that $\alpha$ is a transcendental entire function. We deduce from Lemma 9 and (17) and (18) that $\alpha_{1}$ is a polynomial, which is a contradiction. Thus $\alpha_{1}$ is a polynomial and so is $\beta_{1}$.

So from (12) we get

$$
\begin{aligned}
& {\left[z\left(\left(\alpha_{1}^{\prime}\right)^{k}+P_{k-1}\left(\alpha_{1}^{\prime}\right)\right)+\widetilde{P}_{k-1}\left(\alpha_{1}^{\prime}\right)\right]} \\
& \quad \times\left[\left(\beta_{1}^{\prime}\right)^{k}+Q_{k-1}\left(\beta_{1}^{\prime}\right)\right] e^{3\left(\alpha_{1}(z)+\beta_{1}(z)\right)}=1,
\end{aligned}
$$

where $P_{k-1}\left(\alpha_{1}^{\prime}\right), \widetilde{P}_{k-1}\left(\alpha_{1}^{\prime}\right)$, and $Q_{k-1}\left(\beta_{1}^{\prime}\right)$ are differential polynomials in $\alpha_{1}^{\prime}$ and $\beta_{1}^{\prime}$ of degree at most $k-1$, respectively.

Since $\alpha_{1}^{\prime} \not \equiv 0, \beta_{1}^{\prime} \not \equiv 0$, by (21) we immediately get a contradiction.

Thus $f$ has no zero; similarly, we get that $g$ has no zero. So we have

$$
f(z)=e^{\alpha(z)}, \quad g(z)=e^{\beta(z)},
$$

where $\alpha(z), \beta(z)$ are nonconstant entire functions.

With similar discussion as above, we get that $\alpha+\beta \equiv C$, where $C$ is a constant and $\alpha$ and $\beta$ are both polynomials. 
We deduce from (22) that

$$
\begin{aligned}
& f^{(k)}=\left[\left(\alpha^{\prime}\right)^{k}+P_{k-1}\left(\alpha^{\prime}\right)\right] e^{\alpha}, \\
& g^{(k)}=\left[\left(\beta^{\prime}\right)^{k}+Q_{k-1}\left(\beta^{\prime}\right)\right] e^{\beta},
\end{aligned}
$$

where $P_{k-1}\left(\alpha^{\prime}\right)$ and $Q_{k-1}\left(\beta^{\prime}\right)$ are differential polynomials in $\alpha^{\prime}$ and $\beta^{\prime}$ of degree at most $k-1$, respectively. Thus from (12) we obtain

$$
(-1)^{k}\left(\alpha^{\prime}\right)^{2 k}=z^{2}+\widetilde{P}_{2 k-1}\left(\alpha^{\prime}\right)
$$

If $k \geq 2$, since $\alpha^{\prime}$ is not a constant, $\operatorname{deg}\left(\alpha^{\prime}\right) \geq 1$, by (24) we immediately get a contradiction.

This proves Lemma 10.

\section{Proof of Theorems 1-3}

Since the proof of Theorems 1 and 3 is quite similar to the proof of Theorem 2, here we only need to prove Theorem 2.

Let $F=f^{n} f^{(k)} / z, G=g^{n} g^{(k)} / z, F_{1}=f^{n} f^{(k)}$, and $G_{1}=$ $g^{n} g^{(k)}$. Then $F$ and $G$ share 1 CM and $\infty$ IM.

Suppose that $H \neq \equiv 0$; then $F \not \equiv G$, and $V \not \equiv 0$.

By Lemma 6 we have

$$
\begin{aligned}
T(r, F) \leq & N_{2}\left(r, \frac{1}{F}\right)+N_{2}\left(r, \frac{1}{G}\right)+2 \bar{N}(r, F) \\
& +\bar{N}_{L}(r, F)+\bar{N}_{L}(r, G)+S(r, F)+S(r, G) .
\end{aligned}
$$

So

$$
\begin{aligned}
T\left(r, F_{1}\right) \leq & T(r, F)+\log r \\
\leq & N_{2}\left(r, \frac{1}{F_{1}}\right)+N_{2}\left(r, \frac{1}{G_{1}}\right)+2 \bar{N}\left(r, F_{1}\right) \\
& +\bar{N}_{L}(r, F)+\bar{N}_{L}(r, G)+3 \log r \\
& +S(r, F)+S(r, G) .
\end{aligned}
$$

Obviously,

$$
N\left(r, F_{1}\right)=(n+1) N(r, f)+k \bar{N}(r, f)+S(r, f) .
$$

We have

$$
\begin{aligned}
n m(r, f)= & m\left(r, \frac{F_{1}}{f^{(k)}}\right) \\
\leq & m\left(r, F_{1}\right)+m\left(r, \frac{1}{f^{(k)}}\right)+S(r, f) \\
= & m\left(r, F_{1}\right)+T\left(r, f^{(k)}\right)-N\left(r, \frac{1}{f^{(k)}}\right)+S(r, f) \\
\leq & m\left(r, F_{1}\right)+T(r, f)+k \bar{N}(r, f) \\
& -N\left(r, \frac{1}{f^{(k)}}\right)+S(r, f), \\
& \bar{N}_{L}(r, F)+\bar{N}_{L}(r, G) \leq \bar{N}(r, f) .
\end{aligned}
$$

It follows from (26)-(28) that

$$
\begin{aligned}
(n-1) & T(r, f) \\
\leq & T\left(r, F_{1}\right)-N(r, f)-N\left(r, \frac{1}{f^{(k)}}\right)+S(r, f) \\
\leq & N_{2}\left(r, \frac{1}{F_{1}}\right)+N_{2}\left(r, \frac{1}{G_{1}}\right)+2 \bar{N}(r, F)-N(r, f) \\
& +\bar{N}_{L}(r, F)+\bar{N}_{L}(r, G)+3 \log r-N\left(r, \frac{1}{f^{(k)}}\right) \\
& +S(r, f)+S(r, g) \\
\leq & 2 \bar{N}_{(}\left(r, \frac{1}{f}\right)+2 \bar{N}\left(r, \frac{1}{g}\right)+N\left(r, \frac{1}{g}\right)+(k+1) \bar{N}(r, f) \\
& +\bar{N}_{L}(r, F)+\bar{N}_{L}(r, G)+3 \log r+S(r, f)+S(r, g) \\
\leq & 2 \bar{N}\left(r, \frac{1}{f}\right)+2 \bar{N}\left(r, \frac{1}{g}\right)+N\left(r, \frac{1}{g}\right)+(k+2) \bar{N}(r, f) \\
& +3 \log r+S(r, f)+S(r, g) .
\end{aligned}
$$

Similarly we have

$$
\begin{aligned}
(n-1) & T(r, g) \\
\leq & 2 \bar{N}\left(r, \frac{1}{f}\right)+2 \bar{N}\left(r, \frac{1}{g}\right)+N\left(r, \frac{1}{f}\right)+(k+1) \bar{N}(r, g) \\
& +\bar{N}_{L}(r, F)+\bar{N}_{L}(r, G)+3 \log r+S(r, f)+S(r, g) \\
\leq & 2 \bar{N}\left(r, \frac{1}{f}\right)+2 \bar{N}\left(r, \frac{1}{g}\right)+N\left(r, \frac{1}{g}\right)+(k+2) \bar{N}(r, g) \\
& +3 \log r+S(r, f)+S(r, g) .
\end{aligned}
$$

Combining (29) and (30) gives

$$
\begin{aligned}
(n-1) & (T(r, f)+T(r, g)) \\
\leq & 4\left(\bar{N}\left(r, \frac{1}{f}\right)+\bar{N}\left(r, \frac{1}{g}\right)\right)+N\left(r, \frac{1}{f}\right) \\
& +N\left(r, \frac{1}{g}\right)+(k+1)(\bar{N}(r, f)+\bar{N}(r, g)) \\
& +2\left(\bar{N}_{L}(r, F)+\bar{N}_{L}(r, G)\right)+6 \log r \\
& +S(r, f)+S(r, g) \\
\leq & 5\left(N\left(r, \frac{1}{f}\right)+N\left(r, \frac{1}{g}\right)\right) \\
& +(k+2)(\bar{N}(r, f)+\bar{N}(r, g)) \\
& +6 \log r+S(r, f)+S(r, g) .
\end{aligned}
$$


From (7) and (31) we get

$$
\begin{aligned}
& {[(n-6)(n-k)-4(k+2)](T(r, f)+T(r, g))} \\
& \quad \leq 6(n-k) \log r+S(r, f)+S(r, g) .
\end{aligned}
$$

Case 1. Either $f$ or $g$ is transcendental; from (32) we get a contradiction since $n>k+7>k+6$.

Thus $H \equiv 0$. Similar to the proof of [12, Lemma 3], we obtain

(i) $f^{n} f^{(k)} g^{n} g^{(k)}=z^{2}$, or

(ii) $f^{n} f^{(k)}=g^{n} g^{(k)}$.

By Lemma 10, we get conclusion (2) from (i).

Case 2. Both $f$ and $g$ are rational functions.

If $f$ is a polynomial, so is $g$. We get from (31) that

$$
\begin{aligned}
(2 k+4) \log r+O(1) & \leq(k+2)(T(r, f)+T(r, g)) \\
& \leq 6 \log r+O(1),
\end{aligned}
$$

which implies $k=1, T(r, f)=\log r+O(1)$, and $T(r, g)=$ $\log r+O(1)$.

Set

$$
f(z)=p_{1} z+q_{1}, \quad g(z)=p_{2} z+q_{2},
$$

where $p_{1}, p_{2}, q_{1}, q_{2}$ are constants with $p_{1} p_{2} \neq 0$. By our assumption, we have

$$
f^{n} f^{\prime}-z=d\left(g^{n} g^{\prime}-z\right)
$$

where $d$ is a nonzero constant. By computation we have

$$
\begin{gathered}
p_{1}^{10-j} q_{1}^{j}=d p_{2}^{10-j} q_{2}^{j}, \quad(j=0,1, \ldots, 7,9), \\
9 p_{1}^{2} q_{1}^{8}-1=d\left(9 p_{2}^{2} q_{2}^{8}-1\right),
\end{gathered}
$$

which implies $d=1$ and $f^{n} f^{\prime}=g^{n} g^{\prime}$; thus $f^{n+1}=g^{n+1}+d_{1}$ for a constant $d_{1}$. By the second fundamental theorem we get $d_{1}=0$ and $f \equiv \operatorname{tg}$ for a constant $t$ such that $t^{n+1}=1$.

If $f$ and $g$ are nonpolynomial rational functions, set

$$
f(z)=\frac{R(z)}{T(z)}, \quad g(z)=\frac{U(z)}{V(z)},
$$

where $R(z), T(z), U(z)$, and $V(z)$ are polynomials. Now we discuss three cases as follows.

Case 2.1. Consider $\operatorname{deg} R>\operatorname{deg} T$.

Case 2.1.1. If $\operatorname{deg} U>\operatorname{deg} V$, from (31) we obtain

$$
\begin{aligned}
(k+2) & (m(r, f)+m(r, g)) \\
& +(k+2)(N(r, f)-\bar{N}(r, f)+N(r, g)-\bar{N}(r, g)) \\
\leq & 6 \log r+O(1),
\end{aligned}
$$

which implies that both $f$ and $g$ have only simple poles; thus

$$
\bar{N}_{L}(r, F)+\bar{N}_{L}(r, G)=0 .
$$

From (31) we get

$$
\begin{aligned}
& (k+1)(m(r, f)+m(r, g))+T(r, f)+T(r, g) \\
& \leq 6 \log r+O(1),
\end{aligned}
$$

which is a contradiction since $T(r, f) \geq 2 \log r+O(1)$, $T(r, g) \geq \log r+O(1), m(r, f) \geq \log r+O(1)$, and $m(r, g) \geq$ $\log r+O(1)$.

Case 2.1.2. If $\operatorname{deg} U<\operatorname{deg} V$, from (31) we obtain

$$
\begin{aligned}
8 \log r+O(1) & \leq(k+2) m(r, f)+5 m\left(r, \frac{1}{g}\right) \\
& \leq 6 \log r+O(1),
\end{aligned}
$$

a contradiction.

Case 2.1.3. Consider $\operatorname{deg} U=\operatorname{deg} V$, and $T(r, g)=N(r, g)=$ $\operatorname{deg} U \log r+O(1), m(r, g)=m(r, 1 / g)=O(1)$. It follows from (31) that

$$
\begin{aligned}
(k+2) & m(r, f) \\
& +(k+2)(N(r, f)-\bar{N}(r, f)+N(r, g)-\bar{N}(r, g)) \\
\leq & 6 \log r+O(1) .
\end{aligned}
$$

If $k \geq 2$, then from (42) we get that both $f$ and $g$ have only simple poles; thus

$$
\bar{N}_{L}(r, F)+\bar{N}_{L}(r, G)=0 .
$$

From (31) we get

$$
(k+1) m(r, f)+T(r, f)+T(r, g) \leq 6 \log r+O(1),
$$

which implies that $k=2, T(r, f)=2 \log r+O(1), T(r, g)=$ $\log r+O(1)$, and $m(r, f)=\log r+O(1)$. Set

$$
f(z)=\frac{b_{2} z^{2}+b_{1} z+b_{0}}{z-z_{0}}, \quad g(z)=\frac{c_{1} z+c_{0}}{z-z_{0}},
$$

where $b_{2}, b_{1}, b_{0}, c_{1}, c_{0}$, and $z_{0}$ are constants with $b_{2} c_{1} \neq 0$. Therefore, we have

$$
f^{\prime \prime}=\frac{p_{3}}{\left(z-z_{0}\right)^{3}}, \quad g^{\prime \prime}=\frac{q_{3}}{\left(z-z_{0}\right)^{3}},
$$

where $p_{3}, q_{3}$ are nonzero constants. So $F_{1}-z$ has $2 n$ zeros and $G_{1}-z$ has $n+4$ zeros, which is a contradiction.

If $k=1$, then it follows from (42) that either $f$ or $g$ has only a pole of order at most 2 , and both $f$ and $g$ can not have a pole of order 2 . If $f$ has a pole of order 2 , then $g$ has only simple poles. Thus from (42) we get

$$
2 m(r, f)+T(r, f)+T(r, g) \leq 6 \log r+O(1) .
$$


Obviously, $m(r, f)=\log r+O(1)$. If $T(r, f)=3 \log r+O(1)$, then $T(r, g)=\log r+O(1)$. Set

$$
f(z)=\frac{b_{3} z^{3}+b_{2} z^{2}+b_{1} z+b_{0}}{\left(z-z_{0}\right)^{2}}, \quad g(z)=\frac{c_{1} z+c_{0}}{z-z_{0}},
$$

where $b_{3}, b_{2}, b_{1}, b_{0}, c_{1}, c_{0}$, and $z_{0}$ are constants with $b_{3} c_{1} \neq 0$. Therefore, we have

$$
f^{\prime}=\frac{P(z)}{\left(z-z_{0}\right)^{3}}, \quad g^{\prime}=\frac{Q(z)}{\left(z-z_{0}\right)^{2}},
$$

where $P(z)$ is a polynomial with $\operatorname{deg} P=3 ; Q(z)$ is a nonzero constant. So $F_{1}-z$ has $3 n+3$ zeros and $G_{1}-z$ has $n+3$ zeros, which is a contradiction.

If $T(r, f)=2 \log r+O(1)$, then $T(r, g)=2 \log r+O(1)$. So we have $T(r, f) \geq N(r, f)>N(r, g)=2 \log r+O(1)$, a contradiction.

If $g$ has a pole of order 2 , then $f$ has only simple poles. With similar discussion as above, we get a contradiction.

Therefore, both $f$ and $g$ have only simple poles and we also get (47). Thus $m(r, f)=\log r+O(1)$. If $T(r, f)=$ $3 \log r+O(1)$, then $T(r, g)=\log r+O(1)$; we have $T(r, f)=$ $m(r, f)+N(r, f)=m(r, f)+N(r, g)=2 \log r+O(1)$, which is a contradiction. If $T(r, f)=2 \log r+O(1)$ and $T(r, g)=2 \log r+O(1)$, we also get a contradiction. If $T(r, f)=2 \log r+O(1)$ and $T(r, g)=\log r+O(1)$, then we get (45), which leads to a contradiction.

Case 2.1 has been ruled out.

Case 2.2. Consider $\operatorname{deg} R<\operatorname{deg} T$.

With similar discussion as in Case 2.1, it is easy to get $\operatorname{deg} U=\operatorname{deg} V$. Thus $m(r, f)=m(r, g)=m(r, 1 / g)=O(1)$. Then from (31) we get

$$
\begin{aligned}
& 5 m\left(r, \frac{1}{f}\right) \\
& \quad+(k+2)(N(r, f)-\bar{N}(r, f)+N(r, g)-\bar{N}(r, g)) \\
& \leq 6 \log r+O(1),
\end{aligned}
$$

which implies that both $f$ and $g$ only have simple poles. Moreover, we have $m(r, 1 / f)=\log r+(1)$, and $\bar{N}_{L}(r, F)=$ $\bar{N}_{L}(r, G)=0$. Again from (31) we obtain

$$
\begin{aligned}
7 \log r+O(1) & \leq 5 m\left(r, \frac{1}{f}\right)+T(r, f)+T(r, g) \\
& \leq 6 \log r+O(1),
\end{aligned}
$$

which is a contradiction.

Case 2.2 has been ruled out.

Case 2.3. Thus we have $\operatorname{deg} R=\operatorname{deg} T$. Similarly, we have $\operatorname{deg} U=\operatorname{deg} V$.

It follows from (31) that

$$
\begin{aligned}
& (k+2)(N(r, f)-\bar{N}(r, f)+N(r, g)-\bar{N}(r, g)) \\
& \leq 6 \log r+O(1) .
\end{aligned}
$$

Now we prove that $f$ and $g$ share $\infty \mathrm{CM}$. We discuss two cases below.

Case 2.3.1. If $k \geq 2$, (52) implies that neither $f$ nor $g$ has a pole of order greater than 2; both $f$ and $g$ can not have poles of order 2. Thus only one of $f$ and $g$ may have a pole of order 2.

Suppose that

$$
\begin{aligned}
& f(z)=\frac{b_{l+1} z^{l+1}+b_{l} z^{l}+\cdots+b_{0}}{\left(z-z_{1}\right)^{2}\left(z-z_{2}\right) \cdots\left(z-z_{l}\right)}, \\
& g(z)=\frac{c_{l} z^{l}+c_{l-1} z^{l-1}+\cdots+c_{0}}{\left(z-z_{1}\right)\left(z-z_{2}\right) \cdots\left(z-z_{l}\right)}
\end{aligned}
$$

We deduce from (53) that

$$
\begin{aligned}
F_{1} & =\frac{P_{1}(z)}{\left(z-z_{1}\right)^{2+k}\left(z-z_{2}\right)^{1+k} \cdots\left(z-z_{l}\right)^{1+k}}, \\
G_{1} & =\frac{Q_{1}(z)}{\left(z-z_{1}\right)^{1+k}\left(z-z_{2}\right)^{1+k} \cdots\left(z-z_{l}\right)^{1+k}},
\end{aligned}
$$

where $P_{1}(z), Q_{1}(z)$ are polynomials with $\operatorname{deg} P_{1} \leq(1+k)(l+$ $1)-2 k-1, \operatorname{deg} Q_{1} \leq(1+k) l-2 k-1$. We get that $F_{1}-z$ has $(1+n+k)(l+1)-k+1$ zeros while $G_{1}-z$ has $(1+n+k) l+1$ zeros, which is a contradiction because $F_{1}$ and $G_{1}$ share $z \mathrm{CM}$. Thus $f$ has only simple poles. Similarly, $g$ has only simple poles; thus $f$ and $g$ share $\infty \mathrm{CM}$.

Case 2.3.2. If $k=1$, (31) implies that

$$
\begin{aligned}
2(N & (r, f)-\bar{N}(r, f)+N(r, g)-\bar{N}(r, g)) \\
& +T(r, f)+T(r, g) \\
\leq & 2\left(\bar{N}_{L}(r, F)+\bar{N}_{L}(r, G)\right)+6 \log r+O(1) .
\end{aligned}
$$

If $f$ and $g$ do not share $\infty \mathrm{CM}$, and if $g$ only has simple poles, (55) leads to

$$
\begin{gathered}
2(N(r, f)-\bar{N}(r, f))+T(r, f)+T(r, g) \\
\leq 2 \bar{N}_{L}(r, F)+6 \log r+O(1) .
\end{gathered}
$$

If $f$ has $m$ poles of order $p_{m} \geq 3$, then from (56) we get $m=1$, $T(r, f)=3 \log r+O(1)$, and $T(r, g)=\log r+O(1)$. Set

$$
f(z)=\frac{b_{3} z^{3}+b_{2} z^{2}+b_{1} z+b_{0}}{\left(z-z_{0}\right)^{3}}, \quad g(z)=\frac{c_{1} z+c_{0}}{z-z_{0}} .
$$

With the similar discussion in Case 2.3.1 we get a contradiction.

If $f$ has $m$ poles of order 2 , then from (56) we get $m \leq 2$.

If $m=2$, then $T(r, f)=4 \log r+O(1)$ and $T(r, g)=$ $2 \log r+O(1) \cdot$ Set

$$
\begin{gathered}
f(z)=\frac{b_{4} z^{4}+b_{3} z^{3}+b_{2} z^{2}+b_{1} z+b_{0}}{\left(z-z_{1}\right)^{2}\left(z-z_{2}\right)^{2}}, \\
g(z)=\frac{c_{2} z^{2}+c_{1} z+c_{0}}{\left(z-z_{1}\right)\left(z-z_{2}\right)} .
\end{gathered}
$$


With the similar discussion in Case 2.3.1 we get a contradiction.

If $m=1$, we get (53); with a similar discussion in Case 2.3.1 we get a contradiction.

So $g$ must have multiple poles. Similarly, $f$ must have multiple poles.

From (52) we get that both $f$ and $g$ have one and only one multiple pole and their order is 2 . Since the multiple poles are distinct, then from (55) we get

$$
\begin{aligned}
& f=a \frac{\left(z-a_{1}\right)\left(z-a_{2}\right)\left(z-a_{3}\right)}{\left(z-d_{1}\right)^{2}\left(z-d_{2}\right)}, \\
& g=b \frac{\left(z-b_{1}\right)\left(z-b_{2}\right)\left(z-b_{3}\right)}{\left(z-d_{1}\right)\left(z-d_{2}\right)^{2}},
\end{aligned}
$$

provided that $n=9$, where $a_{j}, b_{j}(j=1,2,3), d_{1}, d_{2}$ are 8 distinct constants and $a, b$ are two nonzero constants. By our assumption, this case has been ruled out. So $f$ and $g$ share $\infty$ CM.

We have

$$
F_{1}=\frac{P_{2}(z)}{Q_{2}(z)}, \quad G_{1}=\frac{P_{3}(z)}{Q_{2}(z)},
$$

where $P_{2}(z), P_{3}(z)$, and $Q_{2}(z)$ are polynomials with $\operatorname{deg} P_{2}<$ $\operatorname{deg} Q_{2}, \operatorname{deg} P_{3}<\operatorname{deg} Q_{2}$. Since $F_{1}$ and $G_{1}$ share $z C M$, we have

$$
P_{2}(z)-z Q_{2}(z)=c\left(P_{3}(z)-z Q_{2}(z)\right),
$$

where $c$ is a nonzero constant; then we get

$$
P_{2}(z)-c P_{3}(z)=(1-c) z Q_{2}(z),
$$

which implies $c=1$ and $P_{2}(z)=P_{3}(z)$. Thus $f^{n} f^{(k)}=g^{n} g^{(k)}$.

This completes the proof of Theorem 2 .

\section{Discussion}

Remark 11. The author can not assert whether (1) really exists because the calculation is rather complicated. The possibility of the existance of (1) is small because the 10 constants must satisfy at least 34 equations. Unfortunately, the author can not prove it. If there exists exceptional case (1), it has its own meaning. It will show that the condition " $n \geq 11$ " of Theorem $\mathrm{B}$ can not be reduced to $n \geq 9$.

Remark 12. One can not get $f \equiv \operatorname{tg}$ for a constant $t$ from $f^{n} f^{(k)}=g^{n} g^{(k)}$. For example, let $f(z)=P(z), g(z)=Q(z)$, where $P(z)$ and $Q(z)$ are polynomials with $\max \{\operatorname{deg} P$, $\operatorname{deg} Q\}<k$. Then $f \not \equiv \operatorname{tg}$ for a constant $t$ but we still have $f^{n} f^{(k)}=g^{n} g^{(k)}$

Problem 4. Can $f^{n} f^{(k)}=g^{n} g^{(k)}$ guarantee $f \equiv \operatorname{tg}$ for a constant $t$ when $f$ and $g$ are transcendental meromorphic functions?

\section{Conflict of Interests}

The author declares that there is no conflict of interests regarding the publication of this paper.

\section{Acknowledgments}

The author would like to thank the referee for valuable suggestions toward this paper. This work is supported by the NSFC (no. 11171184) and the Fundamental Research Funds for the Central Universities (Grant no. 3122013k008).

\section{References}

[1] W. K. Hayman, Meromorphic Functions, Clarendon Press, Oxford, UK, 1964.

[2] C. C. Yang and H. X. Yi, Uniqueness Theory of Meromorphic Functions, Kluwer Academic, Dordrecht, The Netherlands, 2003.

[3] L. Yang, Value Distribution Theory, Springer, Berlin, Germany, 1993.

[4] W. Bergweiler and A. Eremenko, "On the singularities of the inverse to a meromorphic function of finite order," Revista Matemática Iberoamericana, vol. 11, no. 2, pp. 355-373, 1995.

[5] H. H. Chen and M. L. Fang, "On the value distribution of $f^{n} f^{\prime}$," Science in China A: Mathematics, vol. 38, pp. 789-798, 1995.

[6] M. Fang, "A note on a problem of Hayman," Analysis, vol. 20, no. 1, pp. 45-49, 2000.

[7] M. L. Fang and H. L. Qiu, "Meromorphic functions that share fixed-points," Journal of Mathematical Analysis and Applications, vol. 268, no. 2, pp. 426-439, 2002.

[8] J. Xu, F. Lü, and H. X. Yi, "Fixed-points and uniqueness of meromorphic functions," Computers \& Mathematics with Applications, vol. 59, no. 1, pp. 9-17, 2010.

[9] J. L. Zhang, "Uniqueness theorems for entire functions concerning fixed points," Computers \& Mathematics with Applications, vol. 56, no. 12, pp. 3079-3087, 2008.

[10] Y. H. Cao and X. B. Zhang, "Uniqueness of meromorphic functions sharing two values," Journal of Inequalities and Applications, vol. 2012, article 100, 2012.

[11] C. C. Yang, "On deficiencies of differential polynomials II," Mathematische Zeitschrift, vol. 125, pp. 107-112, 1972.

[12] C. C. Yang and X. H. Hua, "Uniqueness and value-sharing of meromorphic functions," Annales Academice Scientiarum Fennica, vol. 22, no. 2, pp. 395-406, 1997.

[13] H. X. Yi, "Meromorphic functions that share three sets," Kodai Mathematical Journal, vol. 20, no. 1, pp. 22-32, 1997. 


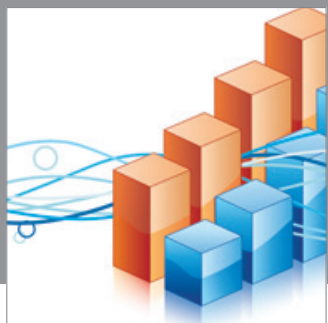

Advances in

Operations Research

mansans

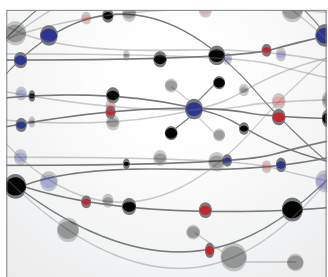

The Scientific World Journal
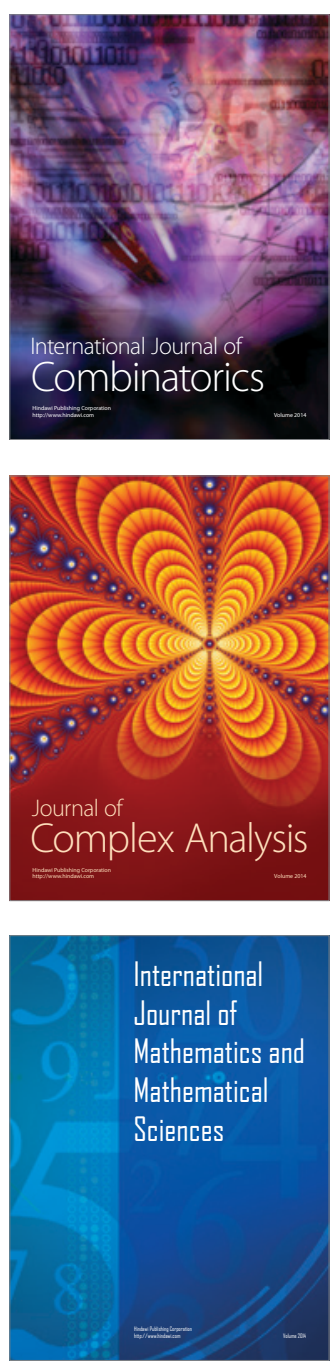
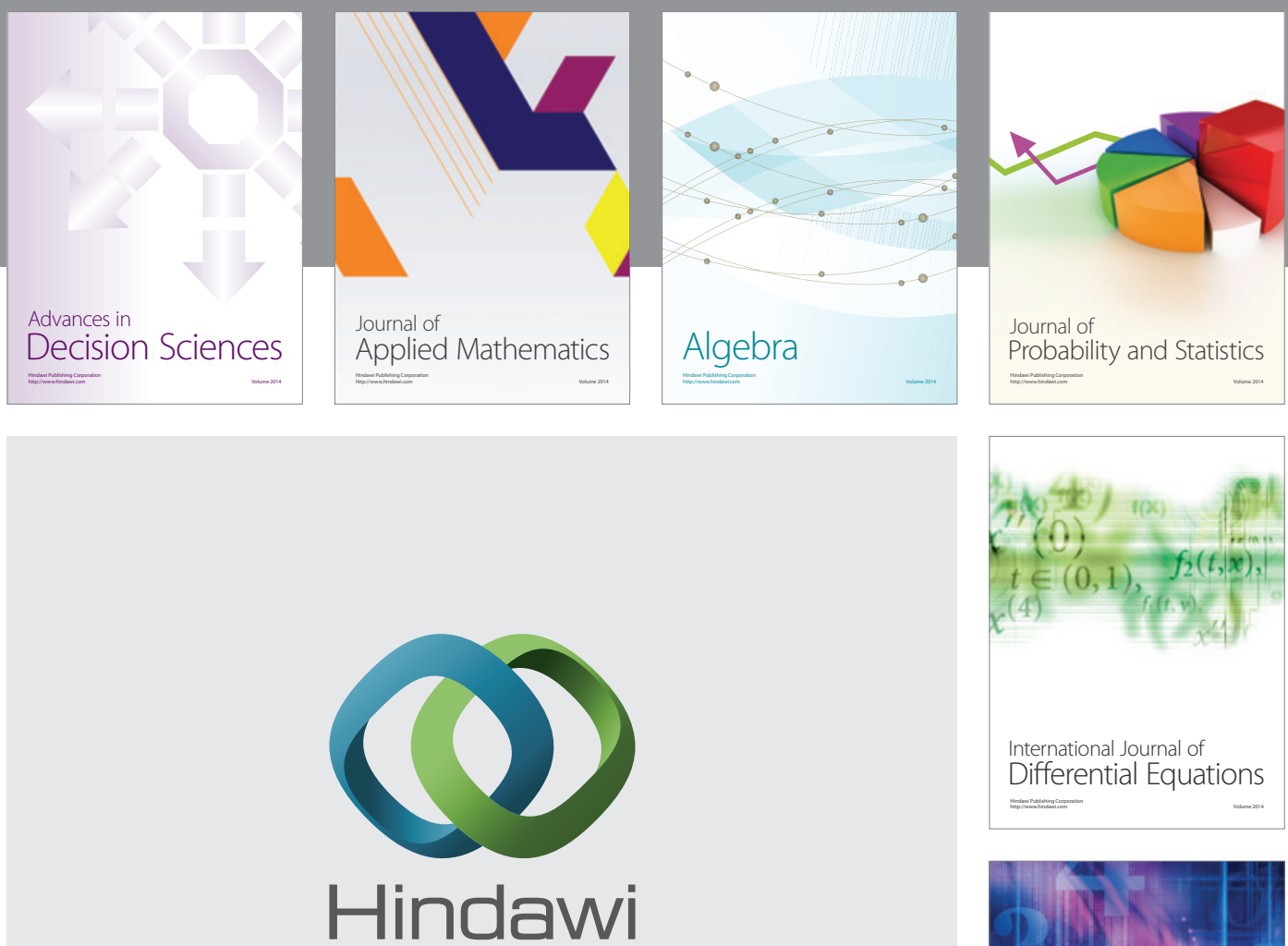

Submit your manuscripts at http://www.hindawi.com
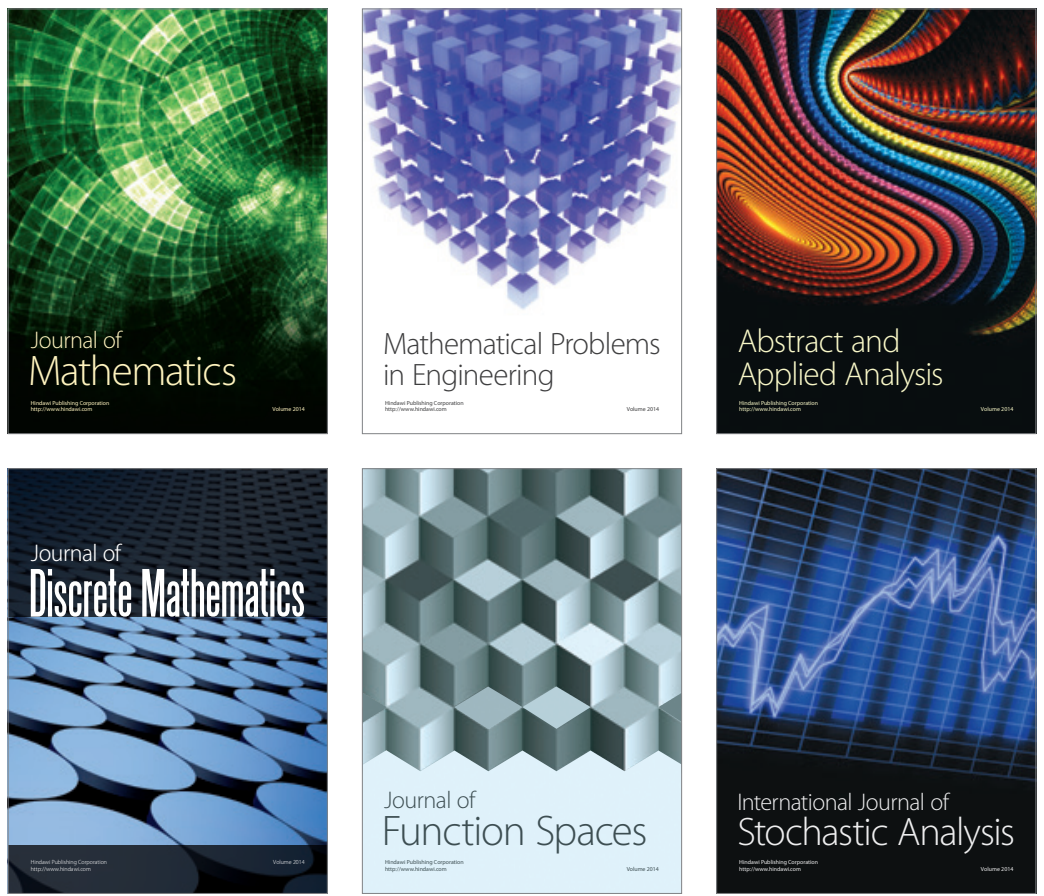

Journal of

Function Spaces

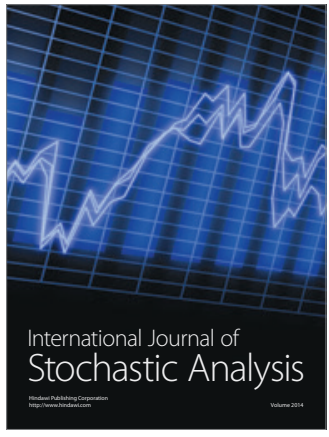

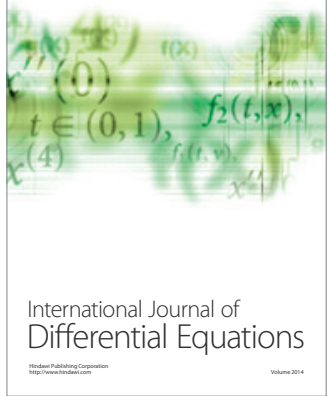
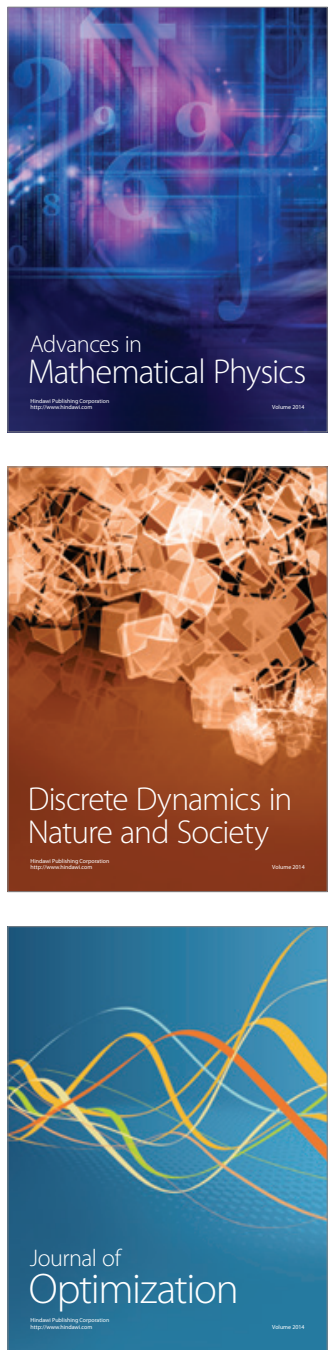